E. Kaier

BASIC-Wegweiser für

IBM PC, PC XT, Portable PC und $\mathrm{PCj}$ 
Mikrocomputer sind Vielzweck-Computer (General Purpose Computer) mit vielfältigen Anwendungsmöglichkeiten wie Textverarbeitung, Datei/Datenbank, Tabellenverarbeitung und Grafik.

Gerade für den Anfänger ist diese Vielfalt oft verwirrend. Hier bietet die Wegweiser-Reihe eine klare und leicht verständliche Orientierungshilfe.

Jeder Band der Wegweiser-Reihe wendet sich an den Benutzer eines bestimmten Mikrocomputers bzw. Programmiersystems mit dem Ziel, Wege zu den grundlegenden Anwendungsmöglichkeiten und damit zum erfolgreichen Einsatz des jeweiligen Computers zu weisen.

Bereits erschienen:

Band 1 BASIC-Wegweiser für den Apple II und kompatible Mikrocomputer

Band 2 MBASIC-Wegweiser für Mikrocomputer unter $\mathrm{CP} / \mathrm{M}$ und MS DOS

Band 3 BASIC-Wegweiser für den Commodore 64

Band 4 BASIC-Wegweiser für IBM PC, PC XT, Portable PC und PCjr

In Vorbereitung:

Band 5 BASIC-Wegweiser für die Commodore-Rechner C 16, C 116, C 264 und C 364

Band 6 BASIC-Wegweiser für MSX-Mikrocomputer

Band 7 Pascal-Wegweiser für Mikrocomputer 
Ekkehard Kaier

\section{BASIC-Wegweiser für IBM PC, PC XT, Portable PC und PCjr}

Mit 111 vollständigen Programmen und zahlreichen Bildern

$\mathbf{V}$

Friedr. Vieweg \& Sohn Braunschweig/Wiesbaden 
Das in diesem Buch enthaltene Programm-Material ist mit keiner Verpflichtung oder Garantie irgendeiner Art verbunden. Der Autor übernimmt infolgedessen keine Verantwortung und wird keine daraus folgende oder sonstige Haftung übernehmen, die auf irgendeine Art aus der Benutzung dieses ProgrammMaterials oder Teilen davon entsteht.

1984

Alle Rechte vorbehalten

๑) Friedr. Vieweg \& Sohn Verlagsgesellschaft mbH, Braunschweig 1984

Die Vervielfältigung und Übertragung einzelner Textabschnitte, Zeichnungen oder Bilder, auch für Zwecke der Unterrichtsgestaltung, gestattet das Urheberrecht nur, wenn sie mit dem Verlag vorher vereinbart wurden. Im Einzelfall muß über die Zahlung einer Gebühr für die Nutzung fremden geistigen Eigentums entschieden werden. Das gilt für die Vervielfältigung durch alle Verfahren einschließlich Speicherung und jede Übertragung auf Papier, Transparente, Filme, Bänder, Platten und andere Medien. Dieser Vermerk umfaßt nicht die in den $\S \S 53$ und 54 URG ausdrücklich erwähnten Ausnahmen.

Umschlaggestaltung: Peter Lenz, Wiesbaden 


\section{Vorwort}

Das Wegweiser-Buch weist Wege zum erfolgreichen Einsatz der IBM Personalcomputer PC, PC XT, Portable PC und PCjr, sofern sie in BASIC programmiert werden.

Das Wegweiser-Buch vermittelt aktuelles Grundlagenwissen zur Datenverarbeitung:

- Was ist Hardware, Software und Firmware?

- Was sind Großcomputer und Mikrocomputer?

- Was sind Datenstrukturen und Programmstrukturen?

- Was sind Betriebssysteme und Anwenderprogramme?

- Was heißt , fertige Programm-Pakete einsetzen'?

- Was umfaßt das eigene Programmieren?

Das Wegweiser-Buch gibt eine erste Benutzungsanleitung:

- Wie startet man die verschiedenen IBM Personalcomputer mit dem Betriebssystem DOS und der Programmiersprache BASIC?

- Wie geht man beim Erstellen eines BASIC-Programmes vor?

- Welche Datentypen und welche Anweisungen enthält BASIC?

- Inwiefern stimmen die IBM-Sprachdialekte BASICA (Advanced BASIC) und Cartridge-BASIC überein?

- Wie setzt man die wichtigsten DOS-Befehle ein?

Es enthält auch einen kompletten Programmierkurs mit einem Grund- und zwei Aufbaukursen:

- Grundkurs:

Programme mit Filge und Auswahlstrukturen, Wiederholungs-(Schleifen)und Unterablaufstrukturen (Unterprogramm, Funktion).

- Aufbaukurs I:

Programmiertechniken, Strings, Ein- und Ausgabe, maschinennahe Programmierung, Programm-Overlay, Arrays sowie Suchen, Sortieren, Mischen und Gruppieren von Daten.

- Aufbaukurs II:

Sequentielle, direkte/random, index-sequentielle und verkettete Organisation einer Datei. Datei mit zeigerverketteter Liste und binärem Baum. Grafik mit niedriger, mittlerer und hoher Auflösung. Programmieren von Spielen und Musik. 
Das Wegweiser-Buch soll die von der IBM gelieferten System-Handbücher keinesfalls ersetzen, sondern ergänzen. In den Handbüchern werden Programmiersprachen (z.B. BASIC-Handbuch), Betriebssysteme (z.B. DOS 2.10 Manual), die technischen Eigenschaften (z.B. Technical Reference), ZusatzGeräte (z.B. Operations Guide) und Software beschrieben. Das WegweiserBuch hingegen beschreibt die Grundlagen der Datenverarbeitung, um sie an zahlreichen BASIC-Anwendungsbeispielen für die IBM Personalcomputer zu veranschaulichen.

Im Wegweiser-Buch sind 111 Programme bzw. Files sowohl als Codierung in BASIC (List) als auch als Ausführung (Run) wiedergegeben und vollständig beschrieben. Die Programmbeispiele werden grafisch als Programmablaufplan (PAP), Struktogramm und Datenflußplan dargestellt.

Die Abschnitte 2 und 3 des Wegweiser-Buches bauen aufeinander auf und sollten in dieser Abfolge gelesen werden. Abschnitt 1 hingegen kann parallel dazu bearbeitet werden.

\begin{tabular}{|l|l|l|}
\hline Abschnitt 1: & & $\begin{array}{l}\text { Abschnitt 2: } \\
\text { Bedienung der } \\
\text { allgemein } \\
\text { IBM Personalcomputer }\end{array}$ \\
\hline & & $\begin{array}{l}\text { Abschnitt 3: } \\
\text { Programmierkurs } \\
\text { in IBM-BASIC } \\
\text { Grundkurs } \\
\text { Aufbaukurs I } \\
\text { Aufbaukurs II }\end{array}$ \\
\hline
\end{tabular}

Für schnelle und eilige PC-Besitzer:

Das Wegweiser-Buch läßt sich auch als Nachschlagewerk benutzen. Aus diesem Grunde wurden das Inhaltsverzeichnis und das Sachwortverzeichnis sehr detailliert aufgegliedert. 


\section{Inhaltsverzeichnis}

1 Computer allgemein $\ldots \ldots \ldots \ldots \ldots \ldots \ldots \ldots \ldots \ldots$

1.1 Computer $=$ Hardware + Software + Firmware $\ldots \ldots \ldots \ldots 2$

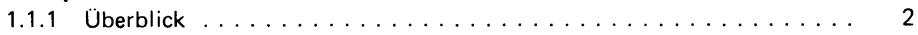

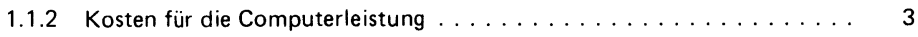

1.1.3 Geschichtliche Entwicklung des Computers . . . . . . . . . . 3

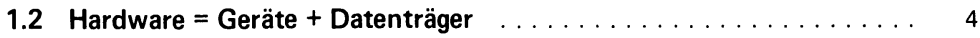

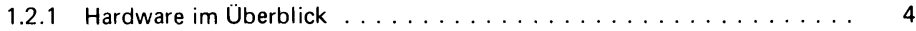

1.2.1.1 Fünf Arten peripherer Geräte bzw. Einheiten . . . . . . . 4

1.2.1.2 Drei Gruppen von Datenträgern ............. 5

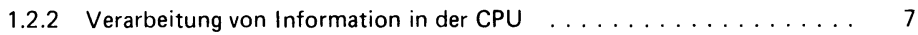

1.2.2.1 Analogie der Datenverarbeitung bei Mensch und Computer . . . 7

1.2.2.2 Computer als speicherprogrammierte Anlage . . . . . . . . 8

1.2.2.3 Computerrechnen im Dual-System Bit für Bit . . . . . . . . . 9

1.2.3 Speicherung von Information intern im Hauptspeicher . . . . . . . . . 9

1.2.3.1 Informationsdarstellung im ASCII und EBCDI-Code . . . . . 10

1.2.3.2 Hexadezimale Darstellung von Zeichen . . . . . . . . . 10

1.2.3.3 Hauptspeicher als RAM und ROM . . . . . . . . . 10

1.2.3.4 Byte als Maßeinheit für die Speicherkapazität $\ldots \ldots \ldots 12$

1.2.4 Speicherung von Information extern auf Datenträgern $\ldots \ldots \ldots \ldots . \ldots . \ldots$

1.2.4.1 Kassette und Magnetband ... . . . . . . . . . . 12

1.2.4.2 Diskette, Winchesterplatte und Magnetplatte . . . . . . . . . 13

1.2.4.3 Klarschriftbeleg als Durckerausgabe . . . . . . . . . . . . 14

1.2.4.4 Schnittstellen als Bindeglieder CPU - Peripherie . . . . . . . 15

1.2.4.5 Back-Up-Systeme zur Datensicherung . . . . . . . . . 16

1.2 .5 Verfahren der Datenerfassung $\ldots \ldots \ldots \ldots \ldots \ldots \ldots \ldots$

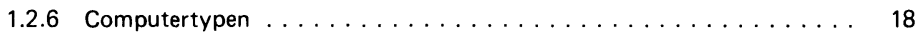

1.2.6.1 System-Konfigurationen für Personal- und Großcomputer . . . 18

1.2.6.2 Eigenschaften von Personalcomputern . . . . . . . . 20

1.2.6.3 Personalcomputer im Computer-Netzwerk . . . . . . . . . 21

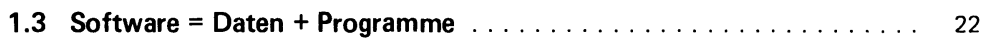

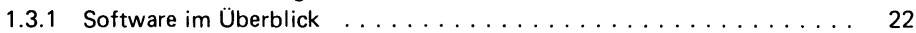

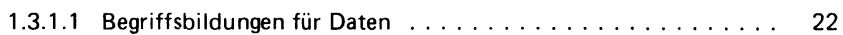

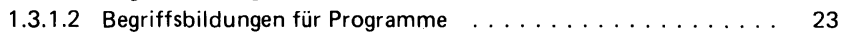

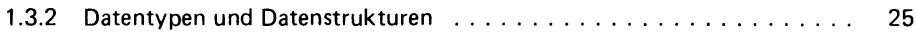

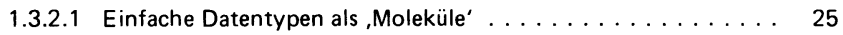

1.3.2.2 Datenstrukturen als strukturierte Datentypen . . . . . . . 26

1.3.2.3 Statische und dynamische Datentypen . . . . . . . . . . 27

1.3.2.4 Vordefinierte und benutzerdefinierte Datentypen . . . . . . . 28

1.3.2.5 Datentypen bei den verschiedenen Programmiersprachen . . . . 28

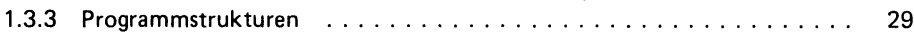

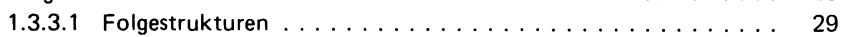

1.3.3.2 Auswahlstrukturen ................... 30

1.3.3.3 Wiederholungsstrukturen ................. 31

1.3.3.4 Unterprogrammstrukturen . . . . . . . . . . . 32

1.3.3.5 Mehrere Strukturen in einem Programm . . . . . . . . . . 32

1.3.4 Daten- und Programmstrukturen als Software-Bausteine . . . . . . . 32

1.3.4.1 Modell des Hauptspeichers RAM als Regalschrank . . . . . . . . 33

1.3.4.2 Daten als Variablen und Konstanten . . . . . . . . . . . 34

1.3.4.3 Programm mit Vereinbarungsteil und Anweisungsteil . . . . . 35

1.3.5 Datei und Datenbank . . . . . . . . . . . . . . 36

1.3.5.1 Zugriffsart, Speicherungsform und Verarbeitungsweise . . . . 37

1.3.5.2 Vier Organisationsformen von Dateien . . . . . . . . . . 40

1.3.5.3 Grundlegende Abläufe auf Dateien . . . . . . . . . . . . 40

1.3.5.4 Datei öffnen, verarbeiten und schließen . . . . . . . . . 42

1.3.5.5 Eine oder mehrere Dateien verarbeiten . . . . . . . . 42

1.3.5.6 Datenbank ........................ 43 


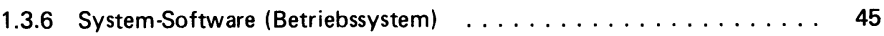

1.3.6.1 Betriebssystem als Firmware (ROM) oder als Software . . . 46

1.3.6.2 Beispiel: Betriebssystem unterstützt Computer-Start . . . . 46

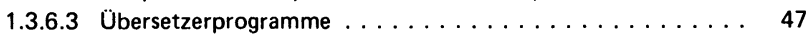

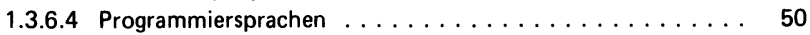

1.3.6.5 Herstellerabhängige und unabhängige Betriebssysteme . . . 52

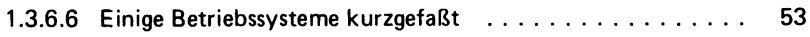

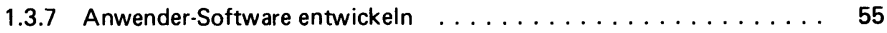

1.3.7.1 Problemanalyse .................. 55

1.3.7.2 Formen zur Darstellung des Lösungsablaufes . . . . . . . 56

1.3.7.3 Programmierung ................ 58

1.3.7.4 Programmiertechniken und Entwurfprinzipien ...... 59

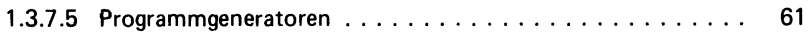

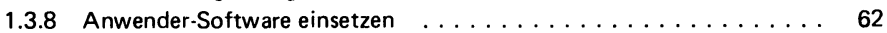

1.3.8.1 Menügesteuerter oder kommandogesteuerter Dialog . . . . 62

1.3.8.2 Einige Programm-Qualitätsmerkmale . . . . . . . . . 62

1.3.8.3 Vier kaufmännische Standard-Programmpakete . . . . . . 63

1.3.8.4 Teillösung und Gesamtlösung im Betrieb . . . . . . . 65

1.3.8.5 Nicht nur am Rande: Spielprogramme . . . . . . . 66

1.4 Firmware $=$ halb Hardware + halb Software $\ldots \ldots \ldots \ldots \ldots$

1.4.1 IC als Integrierter Schaltkreis . . . . . . . . . . . 68

1.4.2 Prinzipieller Aufbau eines Mikrocomputers . . . . . . . . . . . 69

1.4.3 Typen von Mikrocomputern $\ldots \ldots \ldots \ldots \ldots \ldots \ldots \ldots$

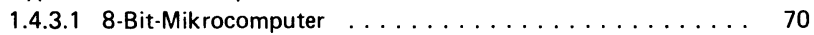

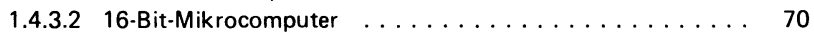

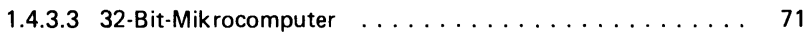

1.4.4 Generationen von Mikroprozessoren . . . . . . . . . . . 72

1.4.5 Mikrocomputer und ihre Mikroprozessoren . . . . . . . . . . . . . . . . . 72

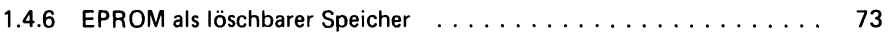

2 Bedienung von IBM PC, PC XT, Portable PC und PCjr . . . . . . . 75

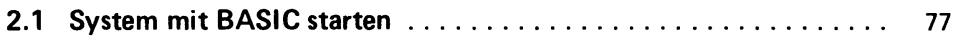

2.1.1 IBM PC, PC XT und Portable PC starten . . . . . . . . . 77

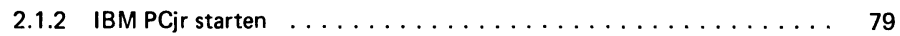

2.1.3 Gegenüberstellung von $\mathrm{PC}$ und $\mathrm{PCjr} \ldots \ldots \ldots \ldots \ldots \ldots$

2.2 Arbeiten im direkten Modus: PC als Tischrechner . . . . . . . 82

2.2.1 Rechnen im direkten Dialog . . . . . . . . . . . . . . . . 82

2.2.2 Editieren des Bildschirminhaltes $\ldots \ldots \ldots \ldots \ldots \ldots$

2.2.3 Text im direkten Dialog $\ldots \ldots \ldots \ldots \ldots \ldots$

2.3 Arbeiten im indirekten Modus: Unser erstes Programm . . . . . . 86

2.3.1 Schritt 1: System mit BASIC starten . . . . . . . . . 86

2.3.2 Schritt 2: Programm Zeile für Zeile eintippen . . . . . . . . 87

2.3.3 Schritt 3: Programm ausführen lassen $\ldots \ldots \ldots \ldots . \ldots \ldots$

2.3.4 Schritt 4: Programm vom RAM auf Diskette speichern ........ 90

2.3.5 Schritt 5: Programm von Diskette in den RAM Iaden ........ 91

2.3.6 Eigentlich Schritt 0: Diskette formatieren . . . . . . . . . 91

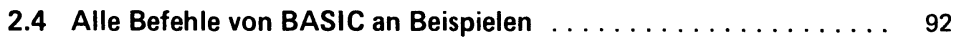

2.4.1 Daten in BASIC . . . . . . . . . . . . . . . 93

2.4.1.1 Konstanten mit Zahlen und Text ... . . . . . . . 93

2.4.1.2 Variablen für einfache Datentypen $\ldots \ldots \ldots \ldots . \ldots . \ldots 9$

2.4.1.3 Variablen für strukturierte Datentypen . . . . . . . . . . . 95

2.4.2 Anweisungen, Funktionen und Operatoren in BASIC . . . . . . 96

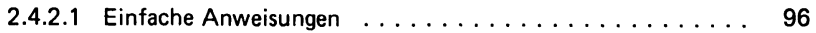

2.4.2.2 Funktionen . . . . . . . . . . . . . . . 102

2.4.2.3 Anweisungen und Funktionen zur Dateiverarbeitung . . . . 105

2.4.3 Operatoren für Rechnen, Vergleich und Logik . . . . . . . . . . . . 108

2.4 .4 BASIC-Dialekte . . . . . . . . . . . . . . . . . . 109

2.4.4.1 Drei BASIC-Dialekte für IBM PC, PC XT und Portable PC .. 109

2.4.4.2 Zwei BASIC-Dialekte für den IBM PCjr . . . . . . . . . 109 
2.5 Grundwissen zum Betriebssystem DOS . . . . . . . . . . 111

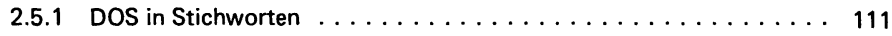

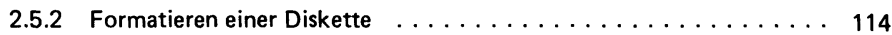

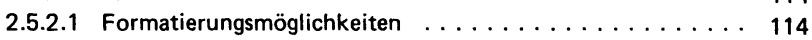

2.5.2.2 Experimentierdiskette einrichten $\ldots \ldots \ldots \ldots \ldots \ldots \ldots 115$

2.5.3 Kopieren einer ganzen Diskette mit DISKCOPY . . . . . . . . . 116

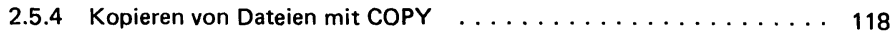

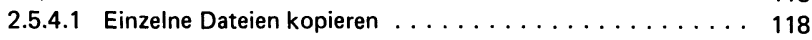

2.5.4.2 Anwendung der Befehle DIR, DEL und REN . . . . . . 119

2.5.4.3 Alle Dateien einer Diskette kopieren . . . . . . . . 121

2.5 .5 Erstellen von Stapel-Dateien . . . . . . . . . . . . . . 122

2.5.5.1 Datei AUTOEXEC.BAT zum automatischen Starten . . . 122

2.5.5.2 Stapel-Datei zum Erzeugen von Anwenderdisketten . . . . 124

2.6 ROM-Cartridge und Diskette als Externspeicher . . . . . . . . 128

2.7 Memory Map der IBM Personal Computer allgemein . . . . . . . . 130

3 Programmierkurs mit IBM-BASIC . . . . . . . . . . . . . . 131

3.1 Grundlegende Programmstrukturen an Beispielen . . . . . . . . 133

3.1.1 Lineare Programme (Folgestrukturen) . . . . . . . . . . . 133

3.1.1.1 Codierung und Ausführungen zu einem Programm . . . . . 133

3.1.1.2 Anweisungsfolge Eingabe - Verarbeitung - Ausgabe . . . 135

3.1.1.3 Übersichtliche Programmgliederung . . . . . . . . 136

3.1.1.4 Programmeingabe und Programmspeicherung . . . . . . . 138

3.1.1.5 Arbeitsschritte zur Programmentwicklung . . . . . . . 139

3.1.2 Programme mit Verzweigungen (Auswahlstrukturen) . . . . . . 141

3.1.2.1 Zweiseitige Auswahl . . . . . . . . . . . . 141

3.1.2.2 Einseitige Auswahl als Sonderfall $\ldots \ldots \ldots \ldots \ldots \ldots \ldots$

3.1.2.3 Mehrseitige Auswahl als Sonderfall . . . . . . . . . 146

3.1.2.4 Fallabfrage ... . . . . . . . . . . . . . . 147

3.1.3 Programme mit Schleifen (Wiederholungsstrukturen) . . . . . . . . 149

3.1.3.1 Abweisende Schleife . . . . . . . . . . . . . . . . 149

3.1.3.2 Nicht-abweisende Schleife . . . . . . . . . . 151

3.1.3.3 Schleife mit Abfrage in der Mitte . . . . . . . . . 152

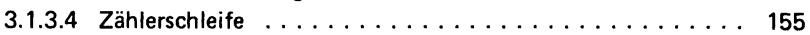

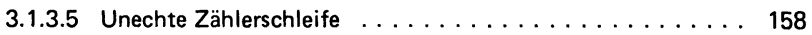

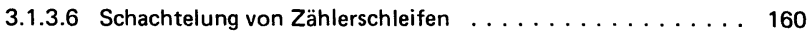

3.1.4 Programm mit Unterprogramm . . . . . . . . . . . . 163

3.1.4.1 Unterprogramme mit GOSUB und RETURN . . . . . . 163

3.1.4.2 Standardfunktionen und selbstdefinierte Funktionen . . . 165

3.2 Programmiertechnik an Beispielen . . . . . . . . . . . . 168

3.2.1 Strukturiert programmieren: Menütechnik $\ldots \ldots \ldots \ldots \ldots$

3.2.2 Wirtschaftlich programmieren: Standardisierung . . . . . . . . 171

3.2.3 Einfach programmieren: Verzweigungstechnik . . . . . . . . . 173

3.2.3.1 Boolesche Variablen und Vergleichsoperationen . . . . . . 173

3.2.3.2 Boolesche Variablen und logische Operationen . . . . . . . 174

3.2.3.3 Vergleich und Logik in der Verzweigungsbedingung . . . . 176

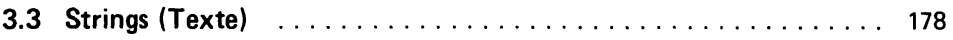

3.3.1 Stringoperationen im Überblick . . . . . . . . . . . . . . 178

3.3.2 Zeichen für Zeichen verarbeiten . . . . . . . . . . . . . . . 179

3.3.3 Datumsangaben verarbeiten . . . . . . . . . . . . . . 186

3.3.4 Teilstrings aufbereiten . . . . . . . . . . . . . . . . . 187

3.3.5 Stringvergleich mit Wildcard-Zeichen . . . . . . . . . . 188

3.3.6 Blocksatz erstellen . . . . . . . . . . . . . . . . . 190

3.3.7 Verschlüsselung zwecks Datenschutz . . . . . . . . . . . . . . 192

3.4 Eingabe und Ausgabe $\ldots \ldots \ldots \ldots \ldots \ldots \ldots \ldots \ldots \ldots$

3.4.1 Bildschirmverwaltung . . . . . . . . . . . . . . . . . 194

3.4.1.1 Farbe, Cursor und Zeichen auf dem Bildschirm . . . . . . . 194

3.4.1.2 Sichere Eingaberoutine . . . . . . . . . . . . . . 197 
3.4.1.3 Bildschirmmaske aufbauen .............. 198

3.4.1.4 Langsame Bildschirmausgabe . . . . . . . . . . 200

3.4.2 Eingabe von ASCII-Code und Zusatzcode . . . . . . . . . . . . . . . 201

3.4.2.1 Zusatzcode über INKEYS eingeben . . . . . . . . . . . 201

3.4.2.2 Unterprogrammaufruf auf Tastendruck . . . . . . . . 204

3.4.2.3 Funktionstasten als Softkeys belegen . . . . . . . . . . 205

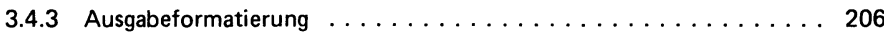

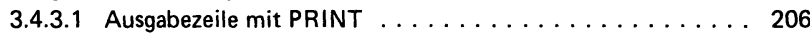

3.4.3.2 Verwendung des Füllstrings . . . . . . . . . . . . 207

3.4.3.3 Zahlen kaufmännisch runden . . . . . . . . . . 208

3.4.3.4 Ausgabezeile mit PRINT USING . . . . . . . . . . 209

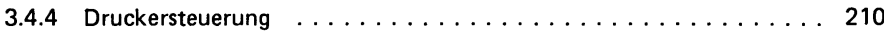

3.5 Maschinennahe Programmierung $\ldots \ldots \ldots \ldots \ldots \ldots \ldots \ldots 213$

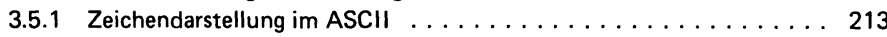

3.5.2 Umwandlung dezimal, binär und hexadezimal . . . . . . . . 215

3.5.3 Daten bitweise verarbeiten . . . . . . . . . . . . . . . 219

3.5.4 Unmittelbarer Zugriff auf Speicherinhalte . . . . . . . . . . . 223

3.5.4.1 Stufe 1: Freien Speicherplatz überprüfen . . . . . . . 223

3.5.4.2 Stufe 2: Speicherplatzinhalte mit PEEK lesen . . . . . . 224

3.5.4.3 Stufe 3: Speicherplatzinhalte mit POKE schreiben . . . . . 225

3.5.4.4 Stufe 4: Aufruf von Maschinenprogrammen mit CALL . . . 227

3.5.4.5 Stufe 5: Maschinenroutinen definieren mit DEF USR . . . 228

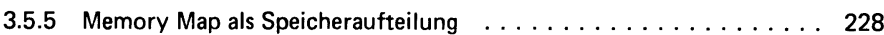

3.5.5.1 Austeilung des gesamten Internspeichers . . . . . . 228

3.5.5.2 Aufbau des BASIC-Speicherbereichs . . . . . . . . 229

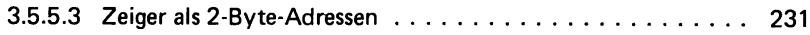

3.5.5.4 Stringspeicher wächst von oben nach unten $\ldots \ldots \ldots \ldots 232$

3.5.6 Speicherung von Daten (Variablen) . . . . . . . . . . . . . 233

3.5.6.1 Variablen stehen hintereinander im Variablenspeicher . . . . 233

3.5.6.2 Format zur Speicherung von Variablen . . . . . . . . 233

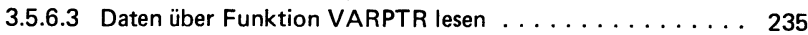

3.5.7 Speicherung von Anweisungen (Programm) . . . . . . . . . . . 239

3.5.7.1 Anweisungen als Token . . . . . . . . . . . . 239

3.5.7.2 Literale im Programmspeicher . . . . . . . . . . . . 242

3.5.7.3 Ein Programm programmiert sich selbst um . . . . . . 242

3.5.8 Garbage Collection als Müllbeseitigung . . . . . . . . . . . . . . . . 244

3.5.9 Programm-Datei und Daten-Datei im ASCII-Format . . . . . . . . . . 246

3.5.9.1 Wörter eines Programms als ASCII-Datei lesen . . . . . . . . 246

3.5.9.2 Wörter einer Datei als ASCII-Datei lesen . . . . . . . . . . . 249

3.5.10 Bildschirminhalt als Binärdatei speichern . . . . . . . . . 250

3.5.10.1 Binärdaten als Speicherabschnitt des RAM schreiben . . . . 250

3.5.10.2 Binärdatei von Diskette in den RAM lesen und zeigen . . . . 252

3.6 Programme überprüfen und Programme verbinden $\ldots \ldots \ldots \ldots 253$

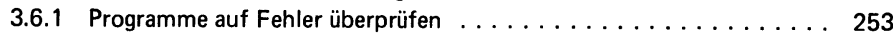

3.6.1.1 Programmtest und Fehlersuche . . . . . . . . . . 253

3.6.1.2 Fehlerbehandlung mit ON ERROR und RESUME . . . . . 254

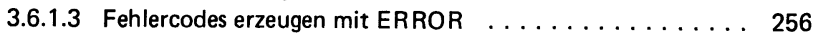

3.6.1.4 Alle Fehlermeldungen nach Nummern geordnet . . . . . . . 256

3.6.2 Programme zu einem Programm-System verbinden . . . . . . . . 258

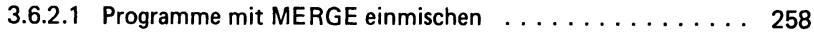

3.6.2.2 Programme mit CHAIN verketten . . . . . . . . 260

3.6.2.3 Gemeinsame Variablen mit COMMON vereinbaren . . . . . 261

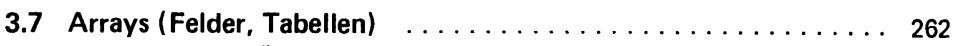

3.7.1 Arrays im Überblick . . . . . . . . . . . . . . . . 262

3.7.2 Eindimensionale Arrays $\ldots \ldots \ldots \ldots \ldots \ldots \ldots \ldots \ldots \ldots \ldots$

3.7.2.1 Numerischer Array zur Speicherung von Zahlen . . . . . . . . 263

3.7.2.2 String-Array zur Speicherung von Text . . . . . . . . . 267

3.7.2.3 Array als Kellerspeicher (Stack) . . . . . . . . . 269 


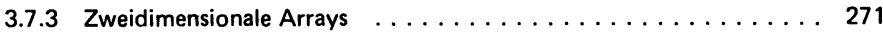

3.7.4 Dreidimensionale Arrays . . . . . . . . . . . . . . . . . 274

3.8 Suchen, Sortieren, Mischen und Gruppieren von Daten . . . . . . . 277

3.8.1 Verfahren im Überblick . . . . . . . . . . . . . . . . . . . 277

3.8 .2 Suchverfahren . . . . . . . . . . . . . . . . . . 277

3.8.2.1 Serielles und sequentielles Suchen . . . . . . . . . . . . 277

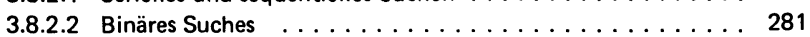

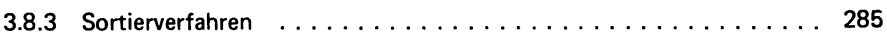

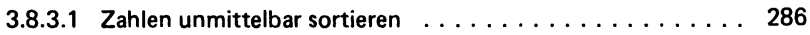

3.8.3.2 Zahlen über Zeiger sortieren . . . . . . . . . . . . . . . . 292

3.8.3.3 Strings unmittelbar sortieren . . . . . . . . . . . 294

3.8.4 Mischen von Arrays . . . . . . . . . . . . . . . . . . . . . 296

3.8.5 Gruppieren von Daten (Gruppenwechsel) . . . . . . . . . . . . . . 297

3.9 Sequentielle Datei (Telephondatei) . . . . . . . . . . . . . . . 298

3.9.1 Menügesteuerte Dateiverwaltung . . . . . . . . . . . . . . . . 299

3.9.2 Dateiweiser Datenverkehr . . . . . . . . . . . . . . . . . . . 299

3.9.3 Verarbeitung von Arrays in den Unterprogrammen . . . . . . . . . 302

3.10 Direktzugriff-Datei (Artikeldatei) . . . . . . . . . . . . . . . . 307

3.10.1 Datei mit konstanter Datensatzlänge . . . . . . . . . . . . . . 308

3.10 .2 Overlay durch Verkettung von Programmen . . . . . . . . . 310

3.10.3 Datensatzweiser Datenverkehr . . . . . . . . . . . . . . 310

3.10.4 Direkte Adressierung des Datensatzes . . . . . . . . . . . . . 317

3.10.5 Indirekte Adressierung des Datensatzes . . . . . . . . . . . . 318

3.11 Index-sequentielle Datei (Kundendatei) $\ldots \ldots \ldots \ldots \ldots \ldots$

3.11.1 Trennung von Datendatei und Indexdatei(en) . . . . . . . . . 319

3.11.2 Zugriff über unsortierte Indexdatei . . . . . . . . . . . . . . . . . . 322

3.11.3 Zugriff über sortierte Indexdatei . . . . . . . . . . . . . . . . . 324

3.11.4 Primärindexdatei und Sekundärindexdateien . . . . . . . . . . . 325

3.11 .5 Voll-Index und Teil-Index . . . . . . . . . . . . . 325

3.12 Gekettete Liste als Linked List (Namendatei) . . . . . . . . . 326

3.12.1 Darstellung einer geketteten Liste . . . . . . . . . . . . . . 327

3.12.2 Erzeugen einer leeren Liste $\ldots \ldots \ldots \ldots \ldots \ldots \ldots \ldots \ldots \ldots \ldots$

3.12.3 Eingeben eines neuen Listenelements . . . . . . . . . . . . . . . . 328

3.12.4 Liste in Sortierfolge oder Speicherfolge ausgeben . . . . . . . . . 331

3.12.5 Gekettete Liste als Datei extern ablegen . . . . . . . . . . . . . . 332

3.12.6 Zusammenfassung der Listenoperationen . . . . . . . . . . 333

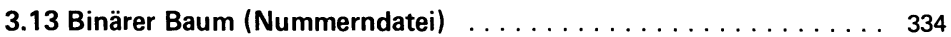

3.13.1 Grafische Darstellung eines Binärbaumes . . . . . . . . . . 334

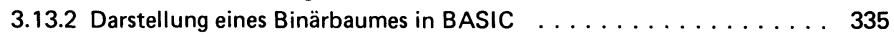

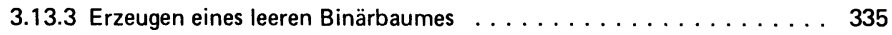

3.13.4 Eingeben von Elementen in den Binärbaum . . . . . . . . 336

3.13 .5 Binärbaum sortiert ausgeben . . . . . . . . . . . . 342

3.13.6 Binärbaum als Datei extern ablegen . . . . . . . . . . . . . . 342

3.14 Verkettete Dateien und Datenbank . . . . . . . . . . . . . . . 343

3.14.1 Externe Verkettung von vier Dateien (Fakturierung) . . . . . . . . . . . 344

3.14.2 Externe wie interne Verkettung von zwei Dateien (Literatur) . . . . . 345

3.15 Grafik ... . . . . . . . . . . . . . . . . . . 347

3.15 .1 Grafik im Überblick . . . . . . . . . . . . . . . . . . 348

3.15.1.1 Text-Grafik und Pixel-Grafik . . . . . . . . . . 348

3.15.1.2 Drei Grafik-Betriebsarten an einem Beispiel . . . . . . . 351

3.15.2 Text-Grafik und Grafik mit niedriger Auflösung . . . . . . . . . 355

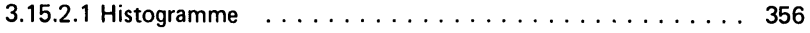

3.15.2.2 Gerade zeichnen . . . . . . . . . . . . . . 361

3.15.2.3 Balkendiagramm . . . . . . . . . . . . . 363

3.15 .3 Grafik mit mittlerer Auflösung . . . . . . . . . . . . . . . . 364

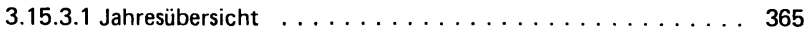

3.15.3.2 Parabeln zeichnen $\ldots \ldots \ldots \ldots \ldots$. . . . . . . 370 
3.15 .4 Grafik mit hoher Auflösung . . . . . . . . . . . . . . . . 373

3.15.4.1 Kreise und Ellipsen $\ldots \ldots \ldots \ldots \ldots \ldots \ldots \ldots \ldots \ldots$

3.15.4.2 Tortendiagramm $\ldots \ldots \ldots \ldots \ldots \ldots \ldots \ldots \ldots \ldots$

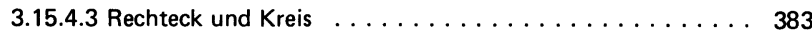

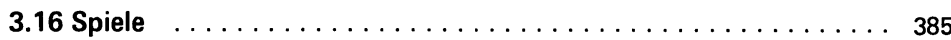

3.16.1 Wortratespiel mit Stringverarbeitung . . . . . . . . . . . . . 385

3.16.2 Biorhythmus als modular aufgebautes Programm . . . . . . . . 386

3.16 .3 Lottozahlen über Index-Array . . . . . . . . . . . . . . . . 389

3.16.4 Lottozahlen durch, Auswahl mit Zurücklegen' . . . . . . . . . . . 391

3.16.5 Elfer-Wette mittels Schleifenschachtelung . . . . . . . . . . . . . 392

3.16.6 Malnehmen als Lernspiel . . . . . . . . . . . . . . . . . . . . . 394

3.16.7 Wandelnder Geist als String-Array . . . . . . . . . . . . . . . . 396

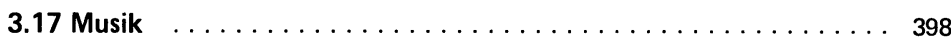

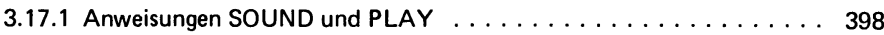

3.17.2 Tonerzeugung an Beispielen . . . . . . . . . . . . . . 400

3.17.3 Programmierung eines Liedes . . . . . . . . . . . . . . . 403

3.17.3.1 Typstring des Liedes festlegen . . . . . . . . . . 404

3.17.3.2 Notenstring des Liedes festlegen . . . . . . . . . . . 406

3.17.3.3 Lied mittels PLAY spielen . . . . . . . . . . . . . . . 407

3.17.4 Noten über Tastatur spielen . . . . . . . . . . . . . . . . . . . . . . . . . . . . . . . . . 407

3.17 .5 Musik im Hintergrund . . . . . . . . . . . . . . . . 408

Programmverzeichnis . . . . . . . . . . . . . . . . . 409

Sachwortverzeichnis $\ldots \ldots \ldots \ldots \ldots \ldots \ldots \ldots \ldots \ldots \ldots$ 